\title{
Enfermedad de Dupuytren, una vista panorámica de nuestro entorno
}

\author{
R. Muñoz Peñin ${ }^{(1)}$, N. LwofF ${ }^{(2)}$,J. M. Arandes Renú ${ }^{(3)}$ \\ (1) Departamento Médico, Pfizer S.L.U. Medical Advisor \\ (2) Departamento Médico, Pfizer S.L.U. Medical Scientific Relations \\ (3) Médico Consultor Servicio COyT. Hospital Clínic. Barcelona
}

\author{
Correspondencia: \\ Dra. Rocío Muñoz Peñin \\ Departamento Médico Pfizer SLU \\ Parque Empresarial La Moraleja \\ Avda, de Europa, 20-B \\ 28108 Madrid \\ Teléfono: +34914909900 \\ Fax: +34914909700
}

La enfermedad de Dupuytren es una condición fibroproliferativa caracterizada por una fascia contráctil que desencadena acortamiento progresivo e irreversible del dedo afecto ${ }^{1}$. El diagnóstico y tratamiento de esta enfermedad en nuestro entorno es un área de interés poco estudiada hasta el momento. Con el objetivo de recoger información relevante al respecto, se elaboró un cuestionario dirigido a especialistas miembros de la Sociedad Española de Cirugía de la Mano. Los resultados nos ofrecen una panorámica general de esta enfermedad y su impacto sobre el Sistema Nacional de Salud. El 60\% de los especialistas diagnostican más de veinte casos anuales, que son intervenidos mayoritariamente mediante fasciectomía parcial. El 83,9\% de los encuestados dice elegir como técnica quirúrgica de elección la fasciectomía parcial como consecuencia de la situación clínica encontrada. Las técnicas con mayor tiempo medio empleado son la fasciectomía radical con una media de 71,6 minutos de quirófano y la dermofasciectomía con 68 minutos. Alrededor de un $50 \%$ de los pacientes van a necesitar rehabilitación. Estos resultados perfilan la situación del diagnóstico y tratamiento de esta patología en España y sus implicaciones sobre el Sistema Nacional de Salud.

Palabras clave: enfermedad de Dupuytren, epidemiología, España, técnicas quirúrgicas, tratamiento.
Dupuytren's disease is a fibroproliferative condition characterized by a contractile fascia that triggers a progressive and irreversible shortening of the affected finger. Diagnosis and treatment of this disease in our environment is an area of interest that has not been fully studied. Regarding this issue, a questionnaire addressed to specialist members of the Sociedad Española de Cirugía de la Mano was developed in order to collect information relevant to this condition. Results offer us an overview of this disease and its impact on the Spanish Health System. Sixty percent of the specialists diagnose more than 20 cases per year, mainly treated through partial fasciectomy. An $83.9 \%$ of those polled answered to choose partial fasciectomy as the surgical technique of choice as a result of the found clinical scenario. The surgical techniques that took the highest mean time to perform are radical fasciectomy and dermofasciectomy, both with 68 minutes. About a $50 \%$ of the patients will need rehabilitation. These results outline the situation of diagnosis and management of this pathology in Spain and its implications on the Spanish Health System.

Key words: Dupuytren's disease, epidemiology, Spain, surgical techniques, treatment/management. 


\section{INTRODUCCIÓN}

\section{Descripción de la patología}

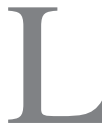

a enfermedad de Dupuytren (ED) es una condición fibroproliferativa caracterizada por el desarrollo de nódulos y cuerdas en la fascia palmar y digital de la mano. Esta patología se define por la presencia de una fascia palmar contráctil que produce un acortamiento y consiguiente alteración de la función de la mano. La afectación es más prevalente en los dedos anular y meñique ${ }^{1}$.

Esta enfermedad se detecta típicamente en la palma de la mano con la aparición de pequeños nódulos que pueden ser palpados y que inicialmente no son demasiado duros. Los nódulos pueden aparecer también en los dedos y, en algunas ocasiones, en otras partes de la mano. La enfermedad de Dupuytren tiende a estabilizarse de manera temporal, pero los nódulos y cuerdas suelen progresar al cabo de los años. A medida que las cuerdas se engrosan, aumentan las fuerzas de contracción que flexionan los dedos afectados hacia la palma de la mano reduciendo su funcionalidad ${ }^{2}$.

La enfermedad de Dupuytren pertenece al grupo de las fibromatosis que incluye también a la fibromatosis plantar o enfermedad de Ledderhose, la fibromatosis del pene o enfermedad de De La Peyronie y la fibromatosis dorsal de las articulaciones interfalángicas proximales, conocida como almohadillas dorsales o nódulos de Garrod $^{3}$.

\section{Etiología y factores hereditarios}

Más de 180 años han transcurrido desde que, en 1831, el Barón Guillaume Dupuytren (17771835) presentó en Paris un caso de retracción permanente de los dedos, analizando los síntomas y demostrando que ni los tendones ni la piel eran la causa de la retracción, enfermedad que recibiría su nombre ${ }^{4,5}$. A pesar del transcurso de tanto tiempo todavía hoy se desconoce la etiología de la enfermedad. No obstante, en la literatura científica hay numerosos datos que asocian un origen genético y hereditario con la aparición de la enfermedad de Dupuytren, mostrándose una expresión variable de la misma según la raza y el sexo de la población estudiada ${ }^{6}$. El vínculo familiar con la patología que se ha encontrado en numerosos estudios dirige la evidencia científica en este sentido. Las observaciones obtenidas en estudios de familias y de gemelos sugieren que la ED tiene un fuerte componente genético y hereditario ${ }^{7}$. Se ha sugerido también que la transmisión de la patología sigue un patrón autosómico dominante de penetrancia incompleta.

\section{Algunos datos epidemiológicos}

Esta enfermedad afecta al 3-6\% de la población general, siendo muy común en el noroeste de Europa $^{8}$. En este sentido, en una revisión sobre la incidencia de la enfermedad en el norte de Alemania realizada por Brenner et al. ${ }^{8}$ el $91,25 \%$ de los pacientes tenían una procedencia germánica «pura», y el 12,5\% tenían antecedentes familiares de la enfermedad. En este estudio, la relación hombre/mujer era de 7 a 1, y la mujer desarrollaba la enfermedad una década más tarde que el hombre, igualándose la tasa hombre/mujer a medida que se envejecía, siendo la prevalencia similar hacia la octava década de la vida. Otro estudio canadiense afirma que la enfermedad de Dupuytren es más frecuente en hombres que en mujeres y se hace sintomática aproximadamente 10 años antes en los hombres que en mujeres 9 . Sin embargo, en general se acepta que dentro de la raza blanca existe una mayor incidencia en la población de origen nórdico y se admite que la enfermedad de Dupuytren es menos frecuente en el sur de Europa, siendo excepcional en los países del Mediterráneo Oriental ${ }^{10}$.

Para la evaluación clínica de esta enfermedad se usa normalmente la clasificación de Tubiana ${ }^{11}$ en la que se proponen una serie de estadios según el grado de deformidad de los dedos afectados (Figura 1).

\section{Tratamiento quirúrgico}

La intervención quirúrgica ha sido la alternativa principal de tratamiento para la ED. En función del criterio del especialista, el tratamiento quirúrgico se suele prescribir cuando los dedos contracturados interfieren en el trascurso de las actividades diarias del paciente ${ }^{12}$.

La cirugía se divide, en líneas generales, en tres técnicas principales, la fasciotomía, la fasciectomía y la dermofasciectomía, que se utili- 


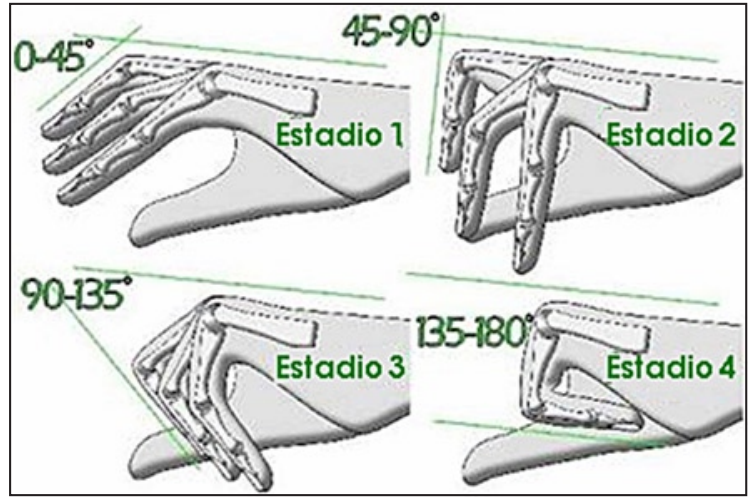

Figura 1. Clasificación de Tubiana ${ }^{11}$.

zarán en función de la situación clínica (Figura 2).

La fasciotomía percutánea corta quirúrgicamente la cuerda fibrosa para liberar la contractura. Se suele considerar adecuada para la enfermedad en estadíos tempranos. La fasciectomía reseca la fascia palmar afectada, incluyendo la cuerda y los nódulos, puede ser parcial o radical. Por último, la dermofasciectomía involucra la eliminación de la fascia palmar afectada y su revestimiento de piel en casos de enfermedad recurrente o en estadío grave de la enfermedad; posteriormente se emplea un injerto de piel para cubrir el defecto cutáneo ${ }^{13}$.

\section{MÉTODO}

Para conocer la situación global de la ED en España se procedió a distribuir un cuestionario de 35 preguntas relevantes entre cirujanos de mano miembros de la Sociedad Española de Cirugía de la Mano (SECMA). Respondieron al cuestionario 87 especialistas distribuidos por el territorio nacional. La encuesta se completó por vía telemática o en papel.

El objetivo final de este cuestionario era recoger información sobre la ED en nuestro entorno referente a diversos asuntos que conforman la situación general de la enfermedad en España. Se procedió a formular preguntas directas al clínico y así registrar, entre otros temas de interés, el número de pacientes con ED que son visitados en su consulta; el lugar de tratamiento de la misma; el tipo de tratamiento más habitual; y aspectos relativos al tratamiento quirúrgico de elección en cada caso, con el objeto de perfilar la situación del diagnóstico y tratamiento de esta patología en España.

El cuestionario constaba de 4 bloques bien diferenciados por área temática:

a) Número de pacientes con ED que visitan, y perfil de los mismos: nueve preguntas relativas a este respecto y a otros asuntos tales como las vías clínicas iniciales de acceso al sistema
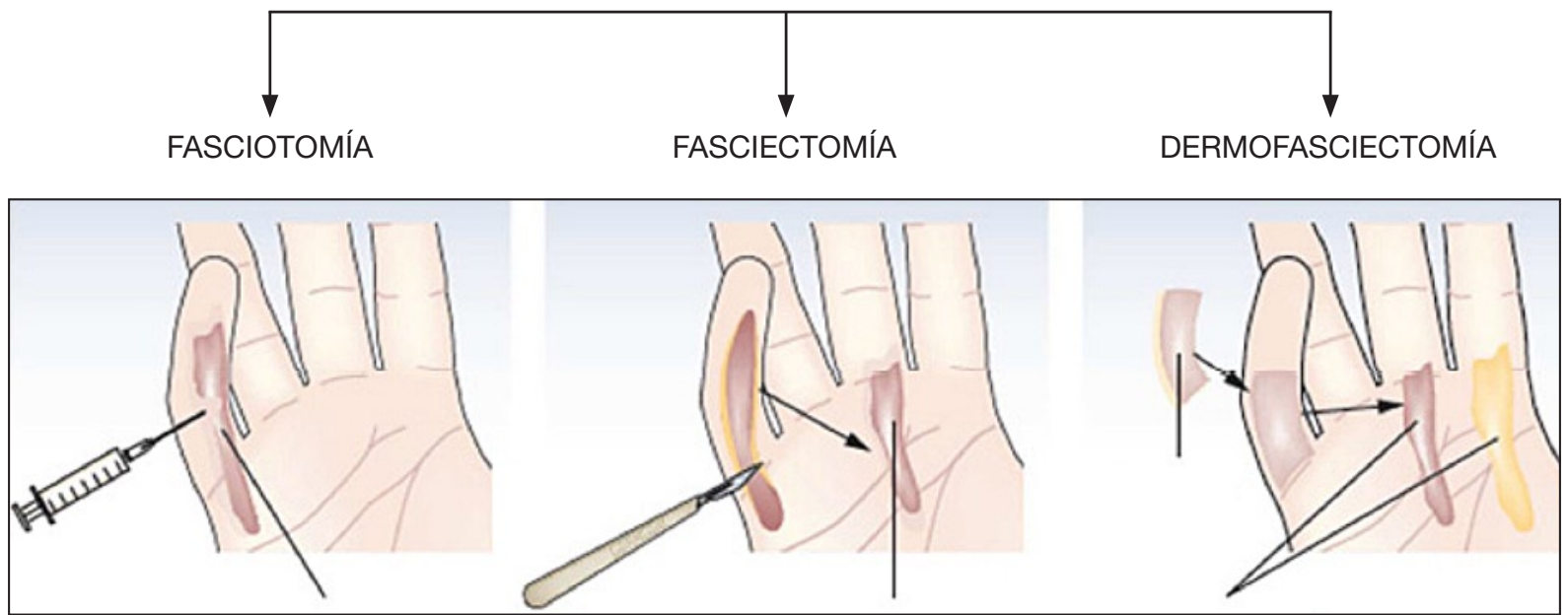

Figura 2. Principales técnicas quirúrgicas. 


\section{TABLA I - FLujo DE PACIENTES}

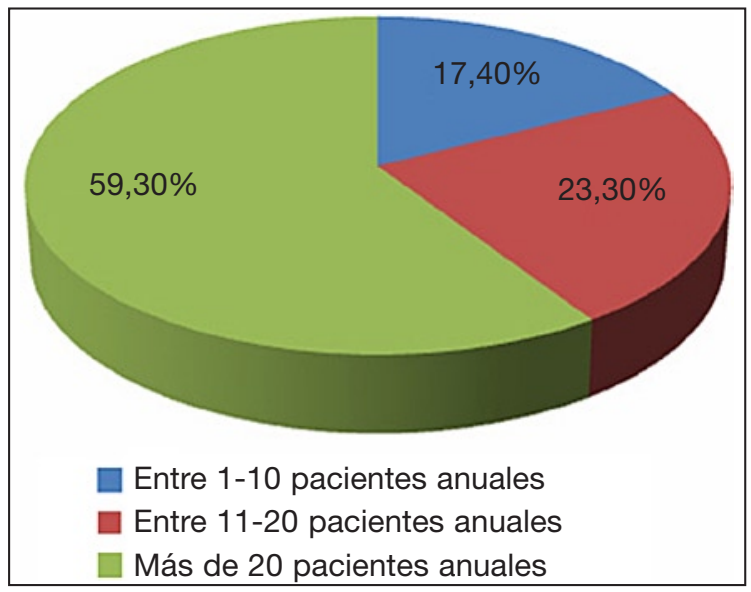

de tratamiento y unidades dónde son tratados y seguidos.

b) Un bloque de catorce preguntas relativas al tratamiento quirúrgico de esta patología: modalidades quirúrgicas más habituales, aspectos específicos y resultados.

c) Otro bloque de siete preguntas referentes a complicaciones, recidivas y rehabilitación de estos pacientes consecuencia del tratamiento quirúrgico de elección en cada caso.

d) Por último, cinco preguntas relativas al consumo de recursos y costes asociados al tratamiento quirúrgico de elección.

\section{RESULTADOS}

Un total de ochenta y siete médicos contestaron al cuestionario por vía telemática (web) o en papel. El 56,3\% de los cuestionarios $(n=49)$ han sido completados en papel, mientras el $43,7 \%$ restante $(n=38)$ por vía web.

Los resultados obtenidos en el primer bloque de preguntas denominado Sección de flujo de pacientes, tiene por objetivo obtener información sobre el perfil del paciente, procedencia del mismo, y características demográficas. Se empieza por preguntar a los clínicos por el número de pacientes que diagnostican anualmente. La respuesta debía facilitarse en escalones cuantitativos de 1-10, 11-20 y más de veinte pacientes.

\section{Tabla II - Procedencia de los} PACIENTES

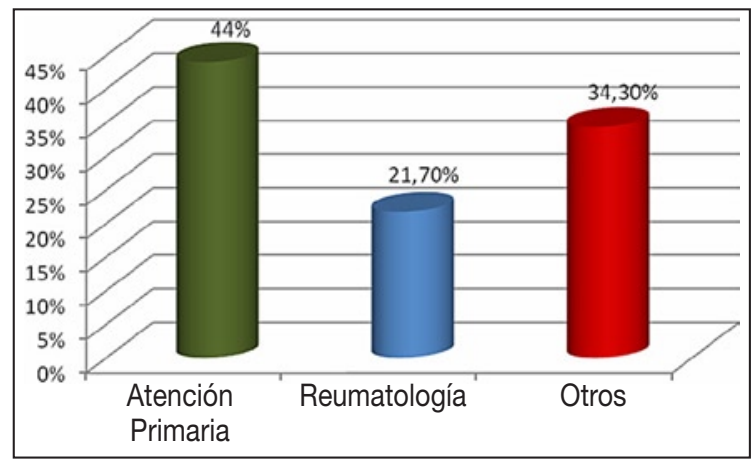

A lo largo de un año, el 59,3\% (n=51) de los especialistas consultados diagnostica ED a más de 20 pacientes, el 23,3\% ( $n=20)$ entre 11 y 20 pacientes y el $17,4 \%$ restante $(n=15)$ entre 1 y 10 (Tabla I).

Cuando los especialistas son consultados sobre cuál es la procedencia clínica de los pacientes que atienden, el $44 \%$ de ellos declara recibirlos desde atención primaria, un $34,3 \%$ de las consultas de reumatología y el $21,7 \%$ restante procede de otras especialidades (traumatología, urgencias, dermatología, rehabilitación etc.) (Tabla II).

La mayoría de los encuestados recibe pacientes mayoritariamente hombres $(53,4 \%$ hombres vs $46,6 \%$ mujeres). El $58,9 \%$ de los pacientes atendidos por los especialistas es mayor de 60 años, y solo un $7,1 \%$ tiene una edad inferior a 40 años.

El 29,2\% de los pacientes diagnosticados tenían una edad comprendida entre 61-65 años, el $28,9 \%$ es mayor de 65 años, el 8,7\% entre $40-50$ años y el 7,1\% una edad inferior a 40 años.

En cuanto a la valoración de los pacientes en el momento del diagnóstico, en el 47,5\% de los casos la enfermedad es unilateral y el 52,5\% bilateral. Además, a la pregunta relacionada con el número de dedos afectados por la condición clínica en el momento del diagnóstico, el 34,7\% de los pacientes recibidos tienen afectado un solo dedo, el 36,0\% dos dedos y el 29,3\% más de dos dedos (Tabla III).

De acuerdo a los criterios de Tubiana ${ }^{11}$, se procedió a cuestionar a los especialistas al res- 


\section{TABLA III - NúMERo de dedos afectados}

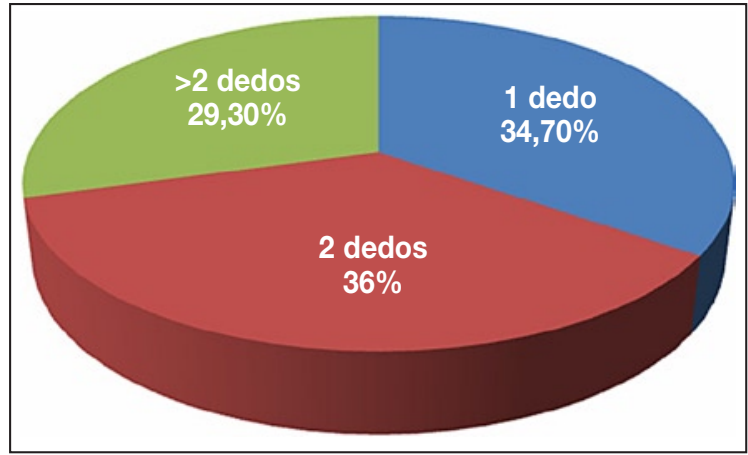

pecto del déficit de extensión. La media de los resultados obtenidos fue muestra de que un elevado porcentaje de pacientes se diagnostican en estadios muy precoces de la enfermedad, de forma que más del $50 \%$ se diagnostica en estadio $\mathrm{N}$ (Tabla IV).

Con respecto a la incapacidad para realizar las actividades normales consecuencia de la condición clínica, el 40,9\% de los encuestados declara que aproximadamente el 10\% de sus pacientes presenta incapacidad a causa de la ED. Únicamente un 4,5\% de los especialistas encuestados la enfermedad relaciona directamente el la incapacidad con la ED. El porcentaje medio de pacientes con incapacidad para realizar las actividades normales a causa de la ED es del 19,7\% de los afectados.

\section{TABLA IV - ESTADIO DE LA ENFERMEDAD EN EL MOMENTO DE DIAGNÓSTICO}

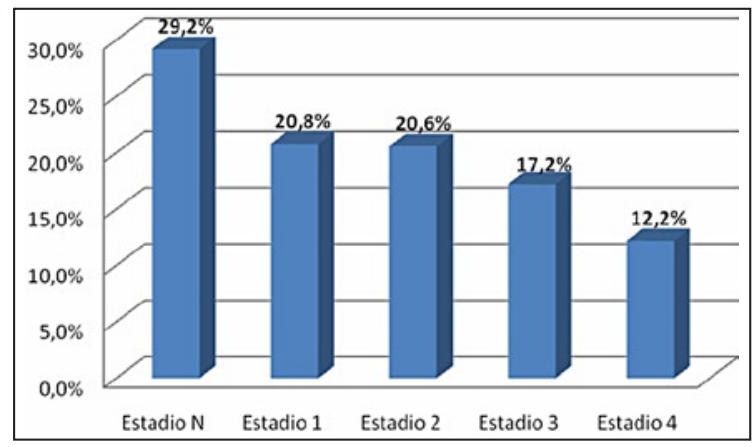

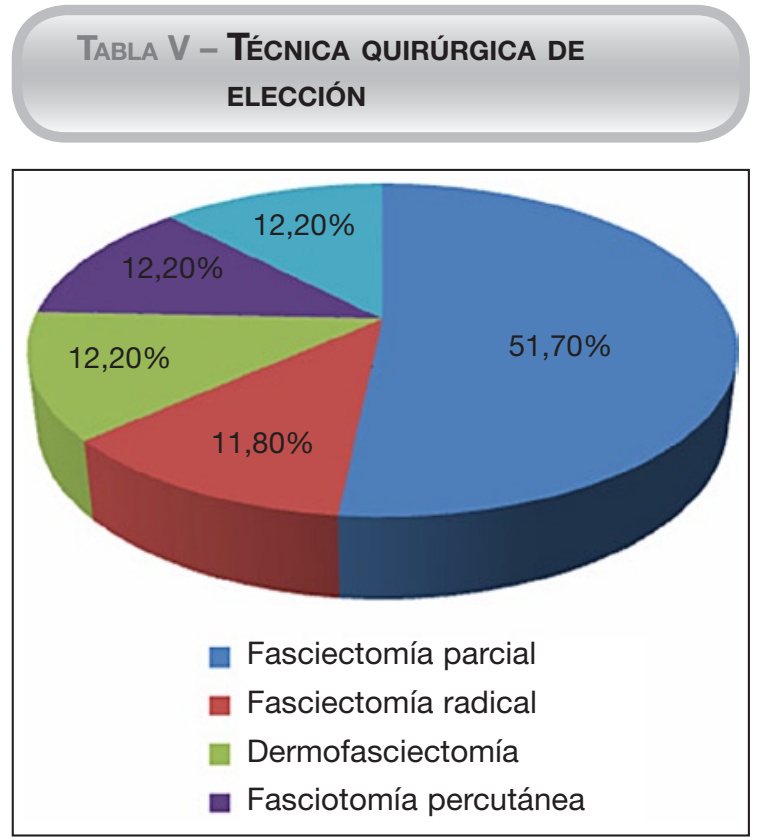

En lo que se refiere al porcentaje de pacientes que son intervenidos quirúrgicamente por esta enfermedad, la percepción de los especialistas consultados es que el $69 \%$ de los pacientes diagnosticados de ED son operados.

El 27,9\% de los médicos encuestados declara que el $80 \%$ de los pacientes diagnosticados de la ED son intervenidos quirúrgicamente, y solo un $7 \%$ de los médicos consultados estiman que se opera al 100\% de los pacientes diagnosticados.

En lo que respecta a la técnica quirúrgica de elección, la más utilizada es la fasciectomía parcial en un $51,7 \%$ de los casos (Tabla V).

El factor determinante para escoger la técnica quirúrgica más adecuada es el grado de afectación del paciente, aunque la edad y las comorbilidades del paciente condicionan, aunque en menor medida, la decisión del cirujano

El tiempo por intervención oscila entre las diferentes técnicas. La fasciectomia radical y la dermofasciectomia requieren más de una hora de quirófano (71,6 minutos y 68 minutos de media respectivamente). La fasciectomia parcial, que es la técnica más empleada, también requiere más de 50 minutos por intervención (53 minutos de media), mientras que la fasciotomia percutánea se realiza en apenas un cuarto de hora $(16,7$ minutos de media) (Tabla VI). 


\section{TABLA VI - TIEMPO MEDIO EMPLEADO EN LAS TÉCNICAS QUIRÚRGICAS (MINUTOS)}

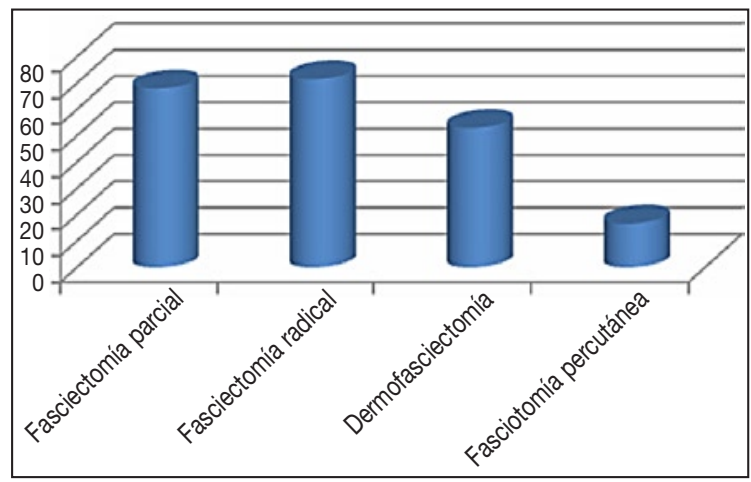

Otro aspecto que se consultó a los especialistas fue la estimación de días de incapacidad para realizar las actividades normales. La fasciectomía radical implica una media de 62,8 días. $\mathrm{La}$ dermofasciectomía requiere igual que la fasciectomía radical más de dos meses. La fasciectomía parcial 48,2 días de media, la fasciectomía limitada 37,9 días y la fasciotomía percutánea 25,9 días.

La ortesis empleada así como el tiempo que se pauta es variable dependiendo del paciente intervenido, del especialista y la técnica escogida.

El 59,9\% de los pacientes sometidos a una fasciectomía parcial necesitarían férula tras la cirugía, un 16,4\% en dermofasciectomía, el 13,4\% en la fasciectomía radical, y un $11,2 \%$ para una fasciotomía percutánea. Además el tiempo medio de utilización de férula tras la cirugía es mayor cuando la técnica quirúrgica empleada ha sido la dermofasciectomía con una media de 56,9 días, 30,5 días en fasciotomía percutánea, 20,5 días en fasciectomía parcial y 23,3 días en fasciectomía radical.

En el Análisis del Sistema Sanitario 2010 para España del Observatorio Europeo de Sistemas y Políticas de Salud (Health Systems In Transition) se reconoce la buena situación del sistema sanitario español en distintos ámbitos evaluados «con la excepción de la información dirigida a los pacientes y la gestión de las listas de espera» ${ }^{14}$. Las listas de espera son, por tanto, un tema de importancia y actualidad así como una medida de la calidad del Sistema Nacional de Salud. Preguntados a este respecto, un $36,1 \%$ de los encuestados declara que existe lista de espera para fasciectomía parcial, y un $17 \%$ dice que hay lista para la dermofasciectomía, un $16,5 \%$ para la fasciotomía percutánea y el 8,2\% para la fasciectomía radical. El tiempo medio de espera para todas las técnicas consideradas en conjunto está en alrededor de los 2-4 meses siendo el mayor tiempo medio de espera para la fasciectomía parcial con 4,4 meses y el tiempo medio mínimo de 2,9 meses para la fasciotomía percutánea.

Preguntados los especialistas sobre la necesidad de rehabilitación en los pacientes intervenidos con las diferentes técnicas quirúrgicas de elección, los resultados muestran que, de media, el $66,2 \%$ de los pacientes a los que se ha realizado una dermofasciectomía necesitan rehabilitación, el 58,2\% de los intervenidos mediante fasciectomías radicales, un $44,60 \%$ de las fasciectomías parciales, el $44,3 \%$ de las fasciotomías percutáneas.

De la información obtenida se desprende que la intervención que requiere un mayor número de días de rehabilitación es la dermofasciectomía con una media de 50,2 días, seguido de la fasciectomía radical 41,9 días, la fasciotomía percutánea con 30,2 días y, por último, la fasciectomía parcial con 29,8 días. Las sesiones de rehabilitación para el 63,2\% de los entrevistados duran más de media hora, mientras que para el $36,8 \%$ restante duran menos de este tiempo.

En cuanto al número de visitas de revisión que los especialistas encuestados necesitan con sus pacientes tras la cirugía, el $86,4 \%$ de los encuestados citan a sus pacientes más de dos veces mientras que el 13,6\% restante lo hace sólo dos veces. De acuerdo a los resultados obtenidos, el tiempo medio entre estas visitas de revisión es de 43,2 días.

La complicación más frecuente asociada a la cirugía que fue reportada por los especialistas, para todas las técnicas empleadas, fue el hematoma. No obstante, de acuerdo con los resultados, el hematoma se produce más frecuentemente en la fasciectomía radical que en el resto de técnicas. La cicatriz hipertrófica, según los cirujanos encuestados, es más frecuente en la dermofas- 
ciectomía con una media del $17 \%$ y la necrosis cutánea también es más frecuente en la dermofasciectomía con una media del 20,2\%.

Con respecto al análisis de las complicaciones comentadas con anterioridad, se preguntó también a los especialistas sobre las técnicas complementarias requeridas para su diagnóstico. El 32,2\% de los encuestados declara que en los casos en los que se presentan complicaciones potoperatorias se requiere una o más pruebas complementarias, siendo las más usadas la ecografía y la microbiología.

En lo que se refiere a los tratamientos en caso de complicaciones postquirúrgicas, el 71,3\% de los encuestados manifiesta que los más habituales son las curas en un $31,7 \%$, el uso de antibióticos en 30,2\%, los injertos en $25,4 \%$ y drenajes en $19 \%$.

Preguntados con respecto a las recidivas en los siguientes años tras la intervención quirúrgica, el $34,1 \%$ de los entrevistados declara que una media del 13,9\% de los pacientes sufrirán recaídas en los próximos años tras haber sido sometidos a fasciectomía parcial. El mayor porcentaje de recidivas se produce tras la fasciotomía percutánea en un $33,1 \%$, mientras que la técnica con menos recidivas, según los clínicos consultados, es la fasciectomía radical con una media de $11,7 \%$.

En cuanto al tiempo de aparición de la recidiva transcurrido tras la intervención, la técnica mediante la cual la recidiva aparece más tarde es la dermofasciectomía con 3,8 años de media, y la que menos tiempo tarda es la fasciotomía percutánea con 1,6 años de media. A este respecto, una media del $46,0 \%$ de las recidivas tras fasciotomía percutánea necesitan de una segunda operación quirúrgica y el 33,8\% de las recidivas por fasciectomía parcial necesitan una segunda intervención.

En el área temática relativa a lo que se denominó en el cuestionario Consumo de recursos derivado del tratamiento quirúrgico de los pacientes con enfermedad de Dupuytren se recogieron los siguientes resultados tras la realización de la encuesta.

Preguntados los especialistas sobre dónde se realizan las intervenciones quirúrgicas por diagnóstico principal ED, las fasciectomías parciales que requieren intervención, el 73,6\% de media son realizadas en hospitales públicos y un 41,6\% en hospitales privados.

Cuestionados sobre el asunto de la realización de cirugía a nivel ambulatorio, de media el 95\% de las fasciotomías percutáneas se realizan a nivel ambulatorio, el 91,3\% de las fasciectomías limitadas, el 89,3\% de las dermofasciectomías, un $77,3 \%$ de las fasciectomías parciales selectivas y un $77,0 \%$ de las fasciectomías radicales.

Una situación que indudablemente repercute sobre el normal transcurso de la vida del paciente y su entorno personal es el número de días de hospitalización. El $61,1 \%$ de las fasciectomías parciales selectivas necesitan ingreso hospitalario, con una media de 1,2 días.

Otro asunto de interés, que requiere de un notable consumo de recursos y tiempo por parte tanto del sistema sanitario como de los pacientes, es la rehabilitación postcirugía. Consultados por este tema, una media del $49,6 \%$ de las fasciectomías parciales requieren de un proceso de rehabilitación postquirúrgico de una duración media de 38,2 días. Por otro lado, el 61,6\% de las dermofasciectomías requieren proceso de rehabilitación postquirúrgico con una duración media de 48,7 días.

En cuanto al personal sanitario especializado necesario para el proceso de rehabilitación del paciente, el 57,3\% requiere un fisioterapeuta en el tratamiento de los pacientes con ED mientras que el 23,6\% necesitarán un rehabilitador y el 19,1\% no necesitará personal sanitario adicional.

\section{CONCLUSIONES}

A lo largo de un año, alrededor del $60 \%$ $(n=51)$ de los especialistas encuestados reconoce diagnosticar más de veinte casos anuales de enfermedad de Dupuytren. El 28,9\% de los pacientes diagnosticados tienen menos de 65 años, y el 53,4\% de los afectados son varones. De acuerdo a los resultados obtenidos en la encuesta, el 52\% de los pacientes tienen afectadas ambas manos por la patología y el $65 \%$ tiene dos dedos implicados o más. Según la encuesta, el porcentaje medio de pacientes con incapacidad para realizar las actividades normales es del $20 \%$ y el porcentaje medio de pacientes operados como consecuencia de la patología es del $80 \%$. 
El 83,9\% de los encuestados dice elegir como técnica quirúrgica de elección la fasciectomía parcial como consecuencia de la situación clínica encontrada. El criterio más utilizado por los encuestados para decidirse a operar a un paciente es, en primer lugar, el grado de afectación y, en segundo lugar, las comorbilidades. Las técnicas con mayor tiempo medio empleado son la fasciectomía radical con una media de 71,6 minutos de quirófano y la dermofasciectomía con $68 \mathrm{mi}$ nutos. El número medio de cirujanos y personal sanitario está entre 1 y 2 para todas las técnicas. La técnica que requiere mayor número medio de días de incapacidad es la fasciectomía radical con 62,8 días de media y la dermofasciectomía con 61,9 días, la fasciectomía parcial con 48,2 días, la fasciectomía limitada con 37,9 días y la fasciotomía percutánea con 25,9 días. El tiempo medio de espera para todas las cirugías esta en torno a los 2-4 meses siendo el mayor tiempo de espera para la fasciectomía parcial con 4,4 meses. Alrededor de un $50 \%$ de los pacientes van a necesitar rehabilitación. La mayoría de los encuestados, notifica citar a sus pacientes tras la cirugía más de dos veces y el tiempo medio entre estas visitas es de 43,2 días.

El 95\% de las fasciotomías percutáneas se realizan a nivel ambulatorio, el $91,3 \%$ de las fasciectomías limitadas, el 89,3\% de las dermo- fasciectomías, un $77,3 \%$ de las fasciectomías parciales selectivas y un $77,0 \%$ de las fasciectomías radicales.

La media de días de hospitalización para este tipo de intervenciones oscila entre los 1,8 días de la dermofasciectomía a los 0,9 de la fasciotomía percutánea. El 71,3\% de los encuestados declara que se requieren tratamientos para las complicaciones postoperatorias.

Los clínicos encuestados declaran que la mayoría de las intervenciones quirúrgicas para el tratamiento de esta patología se realizan en instituciones públicas.

En relación a los recursos empleados, los resultados obtenidos reflejan el impacto que supone, el diagnóstico, tratamiento y seguimiento de los pacientes con ED en España. Las técnicas quirúrgicas más habituales implican un consumo de recursos asociados, que debería ser considerado al valorar las diferentes opciones de tratamiento. En este sentido, es posible que el uso de procedimientos menos invasivos con resultados en eficacia comparables a la cirugía y un perfil de seguridad que facilita la pronta recuperación del paciente, contribuyese a reducir la cantidad de recursos necesarios para el tratamiento de esta patología.

Este estudio ha sido financiado por Pfizer

\section{BIBLIOGRAFÍA}

1 Thurston AJ. Dupuytren's disease. J Bone Joint Surg Br, 2003; 85: 469-77.

2 Larson D, Jerosh-Herold C. Clinical effectiveness of postoperative splinting after surgical release of Dupuytren's contracture: a systematic review. BMC Musculoskelet Disord, 2008; 9: 104.

3 Verheyden CN. The history of Dupuytren's contracture. Clin Plast Surg, 1983; 10: 619-25.

4 Dupuytren G. Rétraction permanente des doigts. Par suite d'une affection de l'aponèvrose palmaire. Leçons orales de la clinique chirurgicale faites à l'Hôtel-Dieu de Paris par M. le Baron Dupuytren, chirurgien en chef, Vol. 1. Paris: Germer BaiIlière, 1832; 2-24.

5 Dupuytren G. Permanent retraction of the fingers, produced by an affection of the palmar fascia. Lancet, 1834; 2: 222-5.

6 Hindocha S, John S, Stanley JK, Watson SJ, Bayat A. The heritability of Dupuytren's disease: familial aggregation and its clinical significance. J Hand Surg Am, 2006; 31: 204-10.

7 Burge, P. Genetics of Dupuytren's disease. Hand Clin, 1999; 15: 63-71.

8 Brenner P, Krause-Bergmann A, Van VH. Die Dupuytren-Kontraktur in Norddeutschland. Epidemiologische Erfassungsstudie anhand von 500
Fällen. Unfallchirurg, 2001; 104: 303-11.

9 Ross DC. Epidemiology of Dupuytren's disease. Hand Clin, 1999; 15: 53-62.

10 Weinstein AL, Haddock NT, Sharma S. Dupuytren's disease in the Hispanic population: a 10-year retrospective review. Plast Reconstr Surg, 2011; 128: 1251-6.

11 Tubiana R. Étude clinique., Évaluation des lésions. En: Tubiana $R(E d)$. Traité de Chirurgie de la Main. Vol 6. Paris: Masson. 1998; 53-66.

12 McGroughter D. Dupuytren's contracture. En: Green D, Hotchkiss R, Pederson W (Ed). Green's Operative Hand Surgery. Vol. 1, 
4th ed, New York: Chruchill Livingstone; 1999; 563-91

13 Bayat A, McGrouther DA. Dupuytren's disease. Surgery, 2006; 24: 373-5.
14 García-Armesto S, AbadíaTaira MB, Durán A, HernándezQuevedo C, Bernal-Delgado E. Spain health system review. Health systems in transition,
Vol. 12, n 4, 2010. http://www. euro.who.int/en/who-we-are/ partners/observatory/healthsystems-in-transition-hit-se ries/countries/spain-hit-2010. 